\title{
Long-distance relationships
}

\author{
Mentoring is important to early career success, but is not always available locally. Several new programs are improving \\ the professional prospects of young scientists by matching them with good mentors elsewhere in the world.
}

G raduate students with mentors are more likely to publish their research and have more interaction with faculty. The importance of good mentorship in cultivating scientific careers is widely appreciated, but for some scientists, developing such a relationship is not so simple. More attention to forming and maintaining these relationships may help to improve retention of underrepresented groups in science.

Mentors can improve scientists' chances of career success and satisfaction at many stages. Formal academic training provides only a small subset of the skills needed for a professional career, which include management ability, financial acuity, conflict resolution (and prevention) and the ability to write grant applications and papers. Mentors can fill the gaps by providing support, inspiration, scientific guidance, help with communication skills and introduction to an international network of scientists. The first mentor for most students is the principal investigator of their undergraduate or graduate lab, and early career professors often look to more senior faculty for advice. Some people, however, cannot find an appropriate mentor so close to home. For example, minorities are less likely to report having a mentor, and women are less likely to be satisfied with the interpersonal interactions they have with their mentors (http://ehrweb.aaas.org/sciMentoring/ MentoringReport.pdf). Given the importance of mentorship to career success, improving the experience might reduce the number of women and minority scientists who leave the laboratory.

One way to increase the likelihood of finding a suitable mentor is to expand the pool of candidates. Though some aspects of the mentoring relationship clearly benefit from face-to-face interactionthere's no substitute for an experienced guide through the political currents of your own department-many do not. The international neuroscience community contains a diverse population of scientists, who could be an important source of mentoring if there were a way to share their experiences beyond local communities. Several initiatives are tapping into this resource, using traditional approaches and emerging technological solutions.

The Society for Neuroscience has two programs to help foster longdistance mentoring relationships: the Neuroscience Scholars program and the Committee on Women in Neuroscience mentoring program. Both programs introduce experienced and young neuroscientists from around the world and provide opportunities for these pairs to make contact in person at the Society's annual meeting. For minorities, the Neuroscience Scholars Program matches undergraduate students, graduate students and postdoctoral fellows with established neuroscientist mentors. For women at all career levels, the Committee on Women in Neuroscience matches interested pairs of women and provides space for them to get together at the annual meeting.

Technology allows mentoring relationships to be developed even when it is not possible to meet in person. One company that takes advantage of this possibility is MentorNet (http://www.mentornet.net). This program matches students and early career researchers with experienced scientists in academia and industry. Each pair exchanges e-mail regularly, with a commitment to corresponding for at least eight months. The convenience and flexibility of the relationship encourage a wide range of participants. The proportion of neuroscientists currently participating in this program is still small, but it continues to grow and is looking for new members in all disciplines.

Another avenue that some neuroscientists are using to make connections is online communities. The Society for Advancement of Chicanos and Native Americans in Science runs an online minority postdoc community that allows members to participate in peer-to-peer mentoring via discussion groups and opportunity listings, and JustGarciaHill is a virtual community for all minorities in science. The American Academy for the Advancement of Science has an online forum for all scientists, along with an advice column directed specifically to minorities in science. These resources offer scientists the opportunity to connect and share experiences where it would be difficult to meet in real life.

A much less formal way of sharing experience is through blogs, where scientists at a variety of career stages are initiating discussions about scientific life. Among the topics recently discussed in cyberspace are work-family balance, applying (and re-applying) for grants and networking at meetings. For example, FemaleScienceProfessor recently posted on whether it is more important to cut back on work hours when children are older, an idea that elicited opinions from both male and female readers. $\mathrm{MsPhD}$, a postdoc in the biomedical sciences, recently wrote on her blog, YoungFemaleScientist, about the frustration induced by the high level of responsibility and low level of short-term payback for postdocs. This post spawned a strong response from other bloggers, including Am I a woman scientist? Many of the blogs respond to one another, creating a network of scientists facing similar issues. The wide range of voices among those writing the blogs, and those commenting on them, can help alleviate the sense of isolation that scientists can experience in the absence of good mentorship.

Mentoring is an important component of career success, but it may not be equally accessible to all scientists. To help plug the 'leaky pipeline', it is crucial to improve resources for connecting with potential mentors. The use of both old-fashioned networking and new technology offers the potential for connections between neuroscientists to extend beyond one's own academic department. These resources represent one method by which access to mentorship can be expanded to a wider audience, potentially improving the retention of scientists from underrepresented groups.

View more background material on Connotea at

http://www.connotea.org/user/NatNeurosci/tag/editorial200710. 